\title{
A 2D+t Feature-preserving Non-local Means Filter for Image Denoising and Improved Detection of Small and Weak Particles
}

Lei Yang ${ }^{1}$

trilithy@gmail.com

Richard Parton ${ }^{2}$

richard.parton@bioch.ox.ac.uk

Graeme Ball $^{2}$

graeme.ball@bioch.ox.ac.uk

Zhen Qiu ${ }^{1}$

zq15@hw.ac.uk

Alan H. Greenaway ${ }^{1}$

a.h.greenaway@hw.ac.uk

Ilan Davis ${ }^{2}$

ilan.davis@bioch.ox.ac.uk

Weiping $\mathrm{Lu}^{1}$

w.lu@hw.ac.uk
${ }^{1}$ Department of Physics

Heriot-Watt University,

Edinburgh, UK

${ }^{2}$ Department of Biochemistry the University of Oxford, Oxford, UK

\begin{abstract}
A feature-preserving non-local means (FP-NLM) filter has been developed recently for denoising images containing small and weak particlelike objects. It explores the commonly used non-local means filter to employ two similarity measurements taken in the original greyscale image and a feature image which measures the particle probability in the original image. In this paper, we report a new approach to image mapping for constructing the feature image by incorporating both spatial and temporal $(2 \mathrm{D}+\mathrm{t})$ characteristics of objects. We present a 2D+t FP-NLM filter based on the improved particle probability image. Experiments show that the new filter can achieve better balance between particle enhancement and background smoothing for images under severe noise contamination and has a greater capability in detecting particles of interest in such environments.
\end{abstract}

\section{Introduction}

Denoising of images containing particle-like objects is an important subject in the field of image processing. In live-cell microscopic imaging, since excitation of fluorescent probes can cause photo-bleaching and photo-toxicity, the light intensity and exposure times that can be used are restricted. The requirement to image fast and in multiple dimensions to 
capture dynamic intracellular events also constrains illumination and exposure regimes. These all result in low signal-to-noise ratio (SNR) fluorescence live-cell images [1-3]. Denoising techniques become a critical tool for quantitative analysis of these images [1-5]. Detection and tracking of small moving targets in synthetic aperture radar (SAR) and infrared (IR) images are other areas with military applications, such as for early warning and long-range defense. These images can also be low SNR, due to the long distance between the targets and imaging devices, unstable radar reflection, IR radiation of the targets and complex backgrounds [6,7]. Noise reduction is an important technique to improve the contrast of small targets for detection and tracking [7,8].

The feature-preserving approach is currently a popular technique for image denoising. A commonly used method for preserving image features is the non-local means (NLM) filter $[9,10]$ in which the filtering strength is determined by the Euclidean distance which measures the difference of pixel grey values between a reference neighborhood and its surrounding neighbors. Patch-based filter (PBF) $[4,11]$ is a state-of-the-art development of NLM filter in which the sizes of the searching windows are adaptively selected. However, for low SNR images containing particle-like features, such as fluorescence live-cell images with mixed Poisson-Gaussian noise contaminations [1-4], the Euclidean distance measured in greyscale images may not be robust for preserving the features [12]. Consequently, NLM filter and other algorithms that are based on this measurement can lead to loss of information and artefacts in denoised images. This calls for new and more robust approaches to feature extraction for image recovery under severe noise contamination.

In a recent study [12], a feature-preserving non-local means (FP-NLM) filter was developed to tackle the above problems. Visually, particle-like features in a single image are manifested as localized concentrations of higher value pixels compared to their immediate surroundings. Such characteristics have been measured by using 2D (XY coordinates) Haar-like features (HLFs) and were adopted for image denoising. Firstly, as depicted in Fig. 1(a-c), three directional HLFs in the 2D space are constructed to measure the local contrast of a small area centred at pixel $\boldsymbol{i}(\boldsymbol{i} \equiv(x, y))$ against its surrounding in a noisy image. Within a given scale, $s$, of a (square) Haar window, the HLFs are defined by

$$
H_{k}(\boldsymbol{i})=\max _{s}\left(H_{k}(\boldsymbol{i}, s)\right) \equiv \max _{s}\left\{M_{U_{k}}(\boldsymbol{i}, s)-M_{V_{k}}(\boldsymbol{i}, s)\right\}
$$

where $M_{U_{k}}$ and $M_{V_{k}}$ are the means of the pixel values in the shaded area, $U_{k}$, and in the white area, $V_{k}$, centred at pixel $\boldsymbol{i}$. As such, $H_{k}$ is the maximum value of local contrasts between two means for all different sizes of the Haar windows. Three HLFs are linearly combined by

$$
\bar{H}(\boldsymbol{i})=\sum_{k=1}^{3} c_{k} H_{k}(\boldsymbol{i})
$$

where $c_{k}$ are the normalized weights. A threshold is then introduced

$$
\bar{H}(\boldsymbol{i}) \geq \lambda
$$

to classify each pixel $\boldsymbol{i}$ into two classes: particle and background. A weak threshold in Eq. 3 is set to ensure that few pixels belonging to particle are wrongly classified. Fig. 1(e) shows an example of HLF image, based on Eq. 2-3, obtained from a synthetic noisecontaminated image Fig. 1(d). The HLF image captures all the particles in the original image as judged by comparing with the known noise free data. The over-estimation of particle class pixels is due to the use of a weak threshold $\lambda$ in the presence of noise. Secondly, the concept of particle probability is introduced. It is defined at each pixel as the ratio of number of pixels that belong to particle and are spatially connected to the total number of pixels in a small region centred at the pixel. Fig. 1(f) shows the particle 
probability image (PPI) of the synthetic image based on the HLF classifications, in which the effect of isolated pixels that are falsely classified as particle has been significantly reduced. The process for constructing the PPI from the original image can be regarded as non-local image mapping. Finally, FP-NLM filter is formulated by extending the commonly used NLM filter to employ two similarity measurements taken in the original image and the PPI. One adapts the other for a greater balance between particle enhancement and background smoothing compared to NLM filter. The denoised image is shown in Fig. 1(g), which recovers all particles seeded in the image.

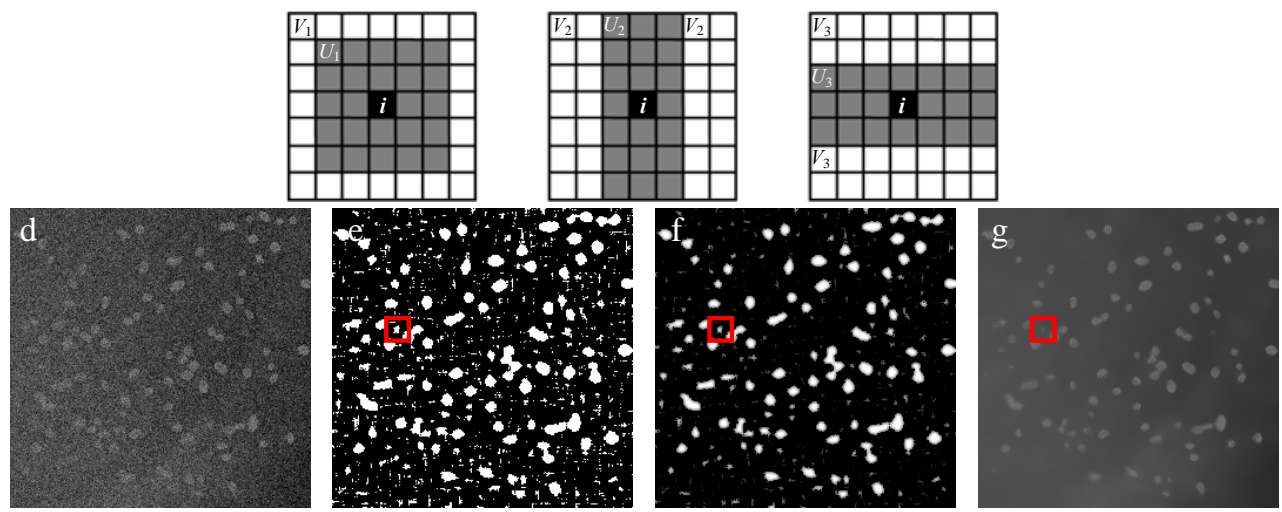

Figure 1: Particle probability image (PPI) mapping. (a-c) 2D Haar windows $H_{k}(\boldsymbol{i}, s)$ $(k=1,2,3)$ proposed in [12]; (d) a synthetic noisy image with Poisson and Gaussian noises (standard deviation $\sigma=20$ for 8 bit data) [4]; (e) binary image showing HLF classifications (the weak threshold (in Eq. 3) $\lambda=6.0$, bright for $\bar{H}(\boldsymbol{i}) \geq \lambda$ and dark for $\bar{H}(\boldsymbol{i})<\lambda$ ); (f) Particle probability image (PPI); (g) 2D FP-NLM filtered result.

The performance of FP-NLM filter depends on the quality of the particle probability images (PPIs) that are constructed by image mapping. Overestimation of particles in these PPIs can become serious when images under investigation are severely noise contaminated and a weak HLF classification threshold is used. This is because in this situation the increased numbers of false HLF classifications at pixel level can give rise to spatially connected clusters, an example of which is shown in the red box in Fig. 1(e). The overestimation leads to the appearance of false particle-like features in PPIs, as illustrated in Fig. 1(f), which in turn results in artefacts in the denoised image, as already starting to appear in Fig. 1(g). The negative effect will become more severe on increasing the noise levels in the images, which can seriously limit the power of FP-NLM filter. In this paper, we will explore a new approach to PPI mapping by extending Haar-like feature extraction from $2 \mathrm{D}(\mathrm{XY})$ space to $2 \mathrm{D}+\mathrm{t}$ space and time domain, where motion continuity in time is used as an unambiguous feature to distinguish particles from noise spikes. We show through experiments on synthetic and real image sequences that the newly proposed $2 \mathrm{D}+\mathrm{t}$ FP-NLM filter can operate under even greater levels of noise contaminations for image denoising and feature recovery.

\section{2D+t feature-preserving non-local means filter}

In this section, we present a new 2D+t feature-preserving non-local means (FP-NLM) filter for denoising a single frame in a time-lapse image sequence. 


\subsection{D+t Haar-like feature-based classification}
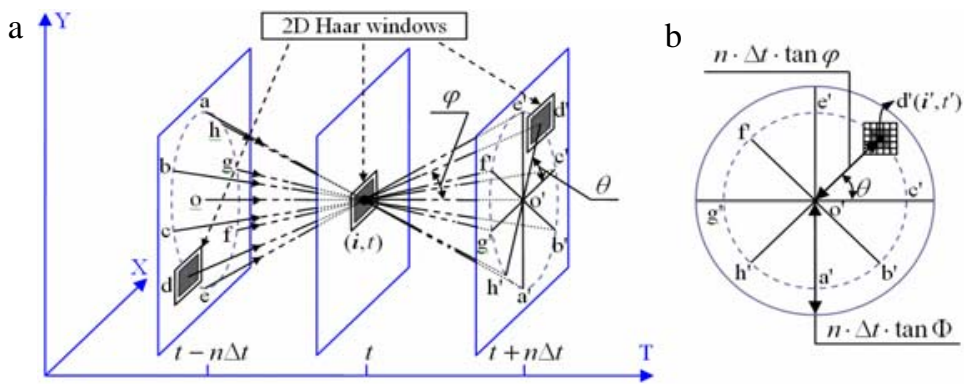

Figure 2: Schematic of 2D+t HLFs. (a) a moving particle in $N=2 n+1$ consecutive frames that pass through pixel $\boldsymbol{i}$ at time $t$, where a-a', b-b', .., o-o' are some of the possible trajectories. The coordinate $\left(x^{\prime}, y^{\prime}\right)$ of pixel $\boldsymbol{i}^{\prime}$ at time $t^{\prime}$ are: $x^{\prime}=x \pm n \cdot \Delta t \cdot \tan \varphi \cdot \cos \theta$ and $y^{\prime}=y \pm n \cdot \Delta t \cdot \tan \varphi \cdot \sin \theta$, where $\Delta t$ is the time interval between frames, $\varphi$ is velocity angle and $\theta$ is direction angle of the motion. (b) parameters at time $t+n \Delta t$.

In object detection and tracking, motion continuity is often used as an unambiguous property for identifying objects of interest from background in an image sequence. We here make use of such motion information of particles to improve the accuracy of Haarlike feature (HLF) classifications. Assuming constant velocity and direction of particle-like objects in neighbouring frames, trajectories of a particle in $N$ consecutive frames that pass through the pixel $\boldsymbol{i}$ at time $t$ are depicted in Fig. 2(a). For a trajectory that has the coordinates $\left(i^{\prime}, t^{\prime}\right)$ at time point $t^{\prime}$, we can apply the 2D Haar windows (Fig. 1(a-c)) to compute local contrasts at this pixel: $H_{k}\left(\boldsymbol{i}^{\prime}, t^{\prime}, s\right)=M_{U_{k}}\left(\boldsymbol{i}^{\prime}, t^{\prime}, s\right)-M_{V_{k}}\left(\boldsymbol{i}^{\prime}, t^{\prime}, s\right)$, where $M_{U_{k}}, M_{V_{k}}$ and $s$ have the same definitions as in Eq. 1. If the trajectory corresponds to that of a true particle, the values of $H_{k}\left(\boldsymbol{i}^{\prime}, t^{\prime}, s\right)$ are usually large (compared to the weak threshold value) and their fluctuations are small over the neighbouring frames. Conversely, if the trajectory is a false one due to noise contamination, the values of $H_{k}\left(\boldsymbol{i}^{\prime}, t^{\prime}, s\right)$ are comparably small and fluctuate more considerably over the neighbouring frames. Using these facts, we define 2D+t HLFs (XYT coordinates) as

$$
H_{k}(\boldsymbol{i}, t)=\max _{s, \theta, \varphi}\left\{\eta(\boldsymbol{i}, \theta, \varphi, t, s) \cdot H_{k}(\boldsymbol{i}, t, s)\right\}
$$

where the coefficient

$$
\eta(\boldsymbol{i}, \theta, \varphi, t, s)=\exp \left(-\frac{1}{N} \cdot \sum_{t^{\prime}=t-n \Delta t}^{t+n \Delta t}\left(\frac{H_{k}\left(\boldsymbol{i}^{\prime}, t^{\prime}, s\right)-\bar{H}_{k}(\boldsymbol{i}, \theta, \varphi, t, s)}{\bar{H}_{k}(\boldsymbol{i}, \theta, \varphi, t, s)}\right)^{2} / 2 \gamma^{2}\right)
$$

measures the consistency of the local contrasts computed for all possible trajectories in $N$ consecutive frames, where $\bar{H}_{k}(i, \theta, \varphi, t, s)=\frac{1}{N} \sum_{t^{\prime}=t-n \Delta t}^{t+n \Delta t} H_{k}\left(\boldsymbol{i}^{\prime}, t^{\prime}, s\right)$ is the mean value, $\theta$ $(\theta \in[0,2 \pi])$ is the direction angle of the trajectory and $\varphi(\varphi \in[0, \Phi])$ is the velocity angle. The angle $\varphi=0$ corresponds to static particles and the maximum angle $\Phi$ to the maximum velocity of particles in the time-lapse sequence. The coefficient $\eta(\boldsymbol{i}, \theta, \varphi, t, s)$ is introduced to penalize false trajectories in which the pixels over neighbouring frames may belong to background or background/particle mixed. The parameter $\gamma$ controls the sensitivity of the penalization. For images of low SNR and with predominately Gaussian 
distribution, we set $\gamma=0.2$, which is around 2 times of the standard deviation of the normalized local contrasts $H_{k}(\boldsymbol{i}, t, s) / \bar{H}_{k}(\boldsymbol{i}, \theta, \varphi, t, s)$ for trajectories corresponding to true particles. Thus, $H_{k}(\boldsymbol{i}, t)$ obtained from Eq. 4 are the consistency weighted maximum values of the local contrasts for all possible scales of the Haar windows ( $s$ ), all possible directions of motion $(\theta)$ and all possible velocities of motion $(\varphi)$. These Haar-like features can in fact be considered as a set of spatiotemporal multi-scale directional filters, which involves the motion information extracted by the simple constant velocity (CV) motion models. The three 2D+t HLFs $H_{k}(\boldsymbol{i}, t)$ can be combined in the same linear way as 2D HLFs (Eq. 2), i.e. $\bar{H}(\boldsymbol{i}, t)=\sum_{k=1}^{3} c_{k} H_{k}(\boldsymbol{i}, t)$. For particles of no preferred shape and orientation, $c_{k}=1 / 3$ is the obvious choice. Each pixel in the image is then classified as particle class if $\bar{H}(\boldsymbol{i}, t) \geq \lambda$ (as Eq. 3 ).

\subsection{Particle probability image}

Because of the finite size of particles, the probability to find a particle depends not only on the HLF in the pixel but also those in its immediate neighbours and whether these pixels are spatially connected. We make use of this non-local property to construct a particle probability image (PPI) from $2 \mathrm{D}+\mathrm{t}$ HLF classifications. It is defined as the ratio

$$
P(\boldsymbol{i})=\left(\Delta N / N_{\text {tot }}\right)_{A_{i}}
$$

where $N_{\text {tot }}$ is the total number of pixels in a given area $A_{\boldsymbol{i}}$ centred at $\boldsymbol{i} ; \Delta N$ is the number of pixels that are classified as particle and spatially connected in $A_{i}$. The size of $A_{i}$ can be chosen as that of the smallest particle in images. The PPI is generated by measuring Eq. 6 in each pixel. Since noise spikes are randomly distributed in space, the effects of overestimation at the pixel level of particle class by HLF classifications can be significantly reduced in the PPI.

\subsection{Implementation of a $2 \mathrm{D}+\mathrm{t}$ feature-preserving non-local means filter}

This section presents a new 2D+t FP-NLM filter that makes use of the improved particle probability mapping from that in 2D domain [12]. For a noisy image $F$, the denoised grey value at pixel $\boldsymbol{i}, F P N L M_{2 D+t}(F)(i)$, is given as the weighted average of all pixel greyscale values in a search window $W_{i}$ centered at $\boldsymbol{i}$

$$
F P N L M_{2 D+t}(F)(\boldsymbol{i})=\sum_{\boldsymbol{j} \in W_{i}} \omega(\boldsymbol{i}, \boldsymbol{j}) F^{\prime}(\boldsymbol{j})
$$

where $F^{\prime}(\boldsymbol{j})=F(\boldsymbol{j})$ if there is no pre-processing on the original image, and the weight is

$$
\omega(\boldsymbol{i}, \boldsymbol{j})=\frac{1}{A(\boldsymbol{i})} \exp \left(-\frac{\left\|\mathbf{V}\left(N_{\boldsymbol{i}}\right)-\mathbf{V}\left(N_{\boldsymbol{j}}\right)\right\|_{2, a}^{2}}{h^{2}}-\frac{\left\|\mathbf{P}\left(N_{\boldsymbol{i}}\right)-\mathbf{P}\left(N_{\boldsymbol{j}}\right)\right\|_{2, a}^{2}}{g^{2}}\right)
$$

where $A(\boldsymbol{i})$ is the normalization constant, $\mathbf{V}\left(N_{i}\right)$ and $\mathbf{P}\left(N_{i}\right)$ respectively are the vectors of the pixel grey values and the particle probability values taken from the neighborhood $N_{i}$ centered at $\boldsymbol{i}$. The first term in Eq. 8 measures similarity of pixel grey values between the 
two neighborhoods $N_{\boldsymbol{i}}$ and $N_{\boldsymbol{j}}$, as in the NLM filter [9], whereas the second term measures the similarity of particle probabilities between the same neighborhoods but taken from the PPI. By appropriately setting the two filtering strength parameters $h$ and $g$, the two measurements can compensate each other to achieve more balanced feature preservation and background smoothing, i.e., the effect of inaccurate similarity measurement in the greyscale image can be reduced by the same measurement in the PPI, whereas overestimation in the PPI will not survive because there is little signature of particles in the greyscale image. Moreover, if we pre-process the image $F(\boldsymbol{j})$ before applying Eq. 7, for example by using the mean filter to smooth the classified background region, the 2D+t FP-NLM filter can significantly reduce the artefacts in background that may be caused by the conventional NLM filtering.

\section{Experimental results}

\subsection{Test on synthetic data}
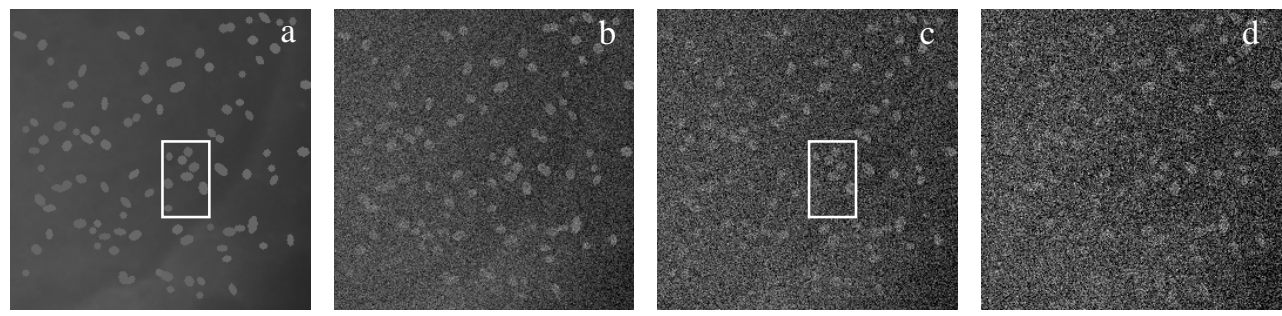

Figure 3. A single frame from a synthetic fluorescence live-cell sequence. (a) noise free image; (b-d) corresponding noisy images of (a) with Poisson and three different levels of Gaussian noises ( $\sigma=20$ (b), 30 (c) and 40 (d)). (b) is the same as Fig. 1(d).

A noise free fluorescence live-cell image sequence, a single frame of which is shown in Fig. 3(a), is firstly synthesized by adding more than 80 particles with different size, shape, intensity and motion parameters above an uneven background. Three noisy image sequences are then constructed by applying a linear model [4] to add Poisson noise and three different levels of Gaussian noises (standard deviations $\sigma=20,30$ and 40 for 8 bit data) to the noise free data. A single frame for each sequence is shown in Fig. 3(b-d).

Test $2 \boldsymbol{D}+\boldsymbol{t} \boldsymbol{H L F s}$ : Since the sizes of particles in the synthetic image sequences are within $5 \times 5$ and $11 \times 11$ pixels, 4 different sizes of Haar windows ( $s=1$ to 4 ) from $9 \times 9$ to $15 \times 15$ are chosen to cover the size range of particles. We set the weak threshold $\lambda$ to be $20 \%$ of the difference of the averaged pixel grey values between a typical particle region and a typical background region [12]. 2D HLF classifications of the two images Fig. 3(c) and (d) are given in Fig. 4(a) and (g), in which an increased presence of false particle pixels is evident on increasing the noise levels. Fig. 4 also shows the classification results of same images using 2D+t HLFs for 3 frames (Fig. 4(b) and (h)) and 5 (Fig. 4(c) and (i)) frames, where the time interval $\Delta t=15$, the maximum velocity angle $\Phi=\pi / 3$ and the resolutions for moving angles $\theta$ and $\varphi$ are $L=36$ and $J=10$. Other parameters are the same as chosen for $2 \mathrm{D}$ HLFs. As seen, though noise is still falsely classified as particles in $2 \mathrm{D}+\mathrm{t}$ HLFs with 3 frames, they intend to be spatially less extended by compared to those by 2D HLF classifications, due to the consistency weighted average over 3 frames (Eq. 4-5). Because of this, a significant improvement to the PPIs is apparent with the $2 \mathrm{D}+\mathrm{t}$ image 
mapping, particularly for images with higher noise levels. We have also computed the results using 2D+t HLFs with 5 frames in Fig. 4. While the false particle pixels are further reduced on increasing the number of frames, true particle pixels can also be misclassified due to severe noise contamination. We find that on balance 2D+t HLFs with 3 frames are the preferred choice for images at the given noise levels.
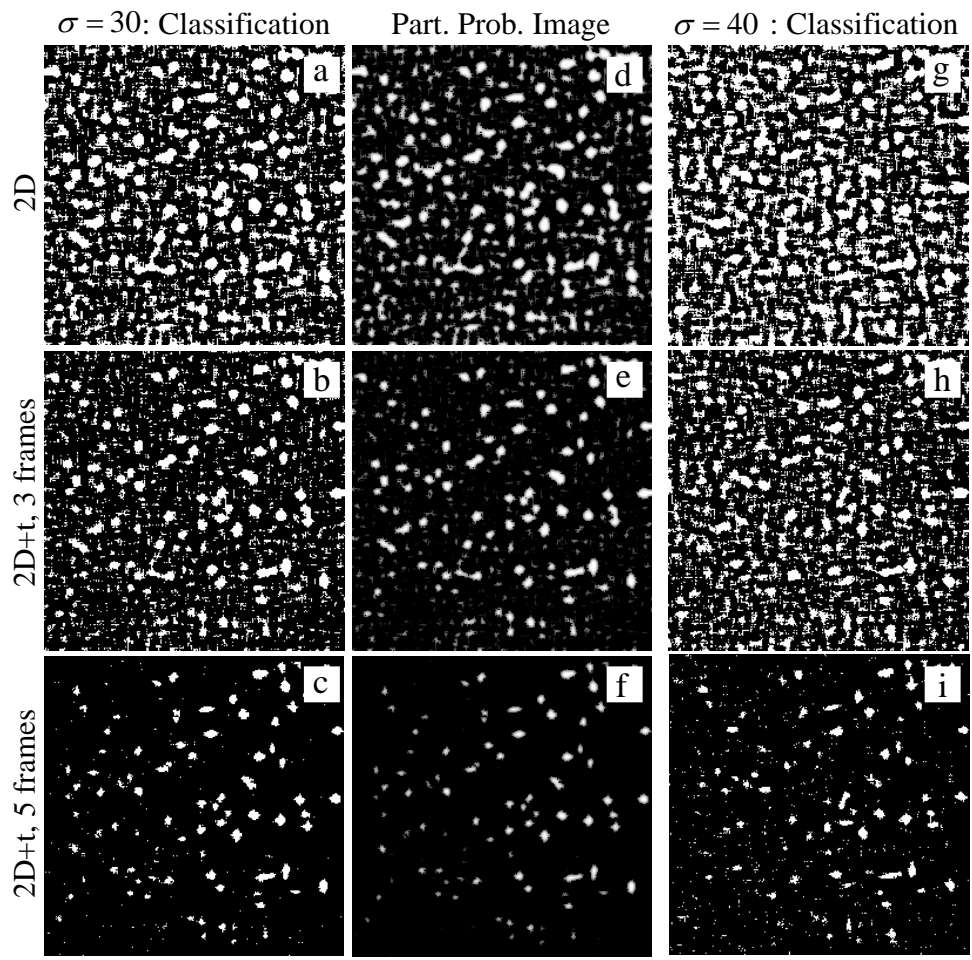

Part. Prob. Image

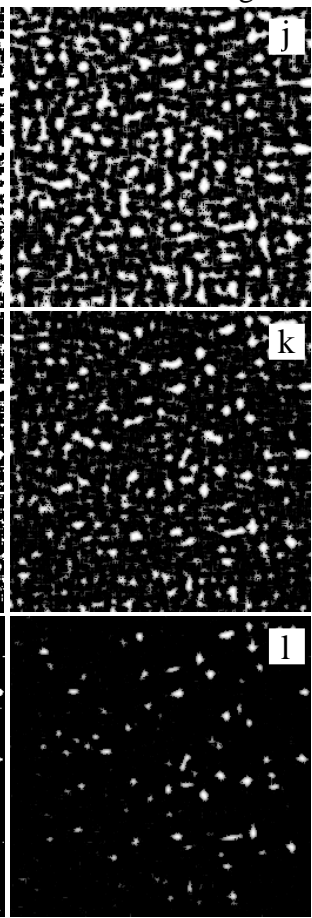

Figure 4. HLF classification and PPI obtained from the raw images Fig. 3(c-d). (a-c) 2D HLFs (a), 2D+t HLFs: 3 frames (b) and 5 frames (c) of Fig. 3(c); (d-f) PPIs corresponding to (a-c); (g-i) 2D HLFs (g), 2D+t HLFs: 3 frames (h) and 5 frames (i) of Fig. 3(d); (j-l) PPIs corresponding to (g-i). Here the threshold $\lambda$ for classification is set to 6.0.

Test $2 D+\boldsymbol{t}$ FP-NLM filter: We have tested the proposed denoising algorithm Eq. 7-8 on the synthetic image sequences. The neighborhood is set to be $7 \times 7$ pixels, the search window is $21 \times 21$ pixels, and the first parameter is $h=\alpha_{1} \cdot \sigma$ and $\alpha_{1}=0.9[9,10]$. The second parameter in 2D FP-NLM filter is set as $g=\sigma / \alpha_{2}$ and $\alpha_{2}=70$ [12]. For 2D+t FPNLM filter, since the PPI gives more accurate particle information and reduced effect of noise spikes, we can increase the second weight in Eq. 8 to further enhance particles while keeping artefacts at minimum level. So we set $g=\sigma / \alpha_{2}$ with $\alpha_{2}=150$. The denoised results of Fig. 3(c) by 2D+t FP-NLM and corresponding 2D FP-NLM filters are shown in Fig. 5(a) and (b), where $F^{\prime}(\boldsymbol{j})$ in both filters are obtained by applying mean filter of $5 \times 5$ pixels to $F(\boldsymbol{j})$ in the classified background regions (Fig. 4(a) and (b)). Particles are preserved very well in Fig. 5(a) and (b), including those of barely visible in the noisy image Fig. 3(c). Moreover, Fig. 5(a) shows an increased particle visibility compared to Fig. 5(b), particularly for weak particles. We have also presented the denoised results of the same image by NLM filter and PBF in Fig. 5(c) and (d). We can see that both 2D+t and 2D FP-NLM filters perform noticeably better than NLM filter and PBF. For comparisons 
of these algorithms in more details, we plot the denoised results (Fig. 5(g-m)) for a small image area given in Fig. 5(f), taken from the squared window in Fig. 3(c). As seen, oversmoothing by NLM filter and PBF on weak particles is evident (Fig. 5(l-m)). 2D+t FPNLM filter (Fig. 5(h)) performs better on enhancing the signal intensity of particles than 2D FP-NLM filter (Fig. 5(j)) thanks to a larger filtering strength for particle preserving (parameter $\alpha_{2}$ ), particularly for weak particles (compare the particle areas marked by squares in Fig. 5(h) and (j)). We note that the higher signal intensity can also be obtained by increasing $\alpha_{2}$ for the 2D FP-NLM filter (compare the same square-enclosed particle areas in Fig. 5(j) and (k)), but false classifications by 2D HLFs will be also enhanced as artifacts (compare the background areas marked by circles in Fig. 5(i) and (k)). Thus, the capability of the 2D FP-NLM filter is limited compared to 2D+t FP-NLM filter. To quantify image fidelity of the denoised results, we calculate the peak signal-to-noise ratio (PSNR) [11,12] for the denoised results of images Fig. 3(b-d) using the above filters and other commonly used algorithms. The latter includes nonlinear anisotropic diffusion (NAD) [13], total variation (TV) minimization [14], Wiener filtering (WF), bilateral filtering (BF) [15], all the parameters for these filters have the same values as in [12]. The results in Table 1 show that the 2D and 2D+t FP-NLM filters generally achieve a higher PSNR compared with other filters, and the 2D+t FP-NLM filter has a better capability in restoring images with more severe noise contamination ( $\sigma=30$ to 40 ).
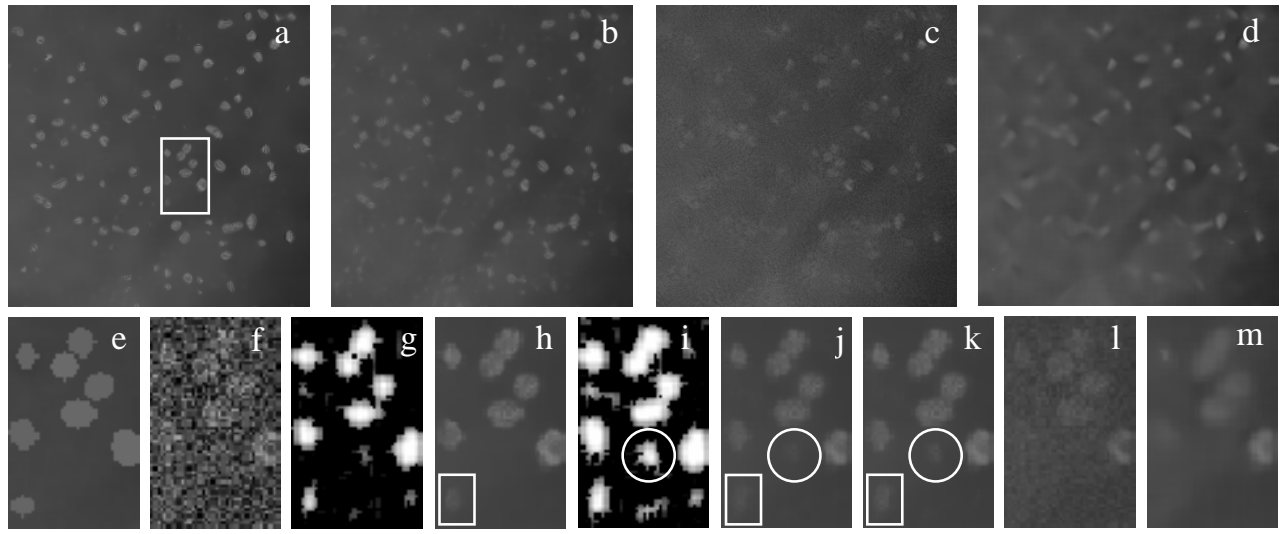

Figure 5. Denoising results on Fig. 3(c). (a) 2D+t FP-NLM filter (3 frames); (b) 2D FPNLM filter; (c) NLM; (d) PBF; (e) a selected noise free region and (f) the corresponding noisy region taken from Fig. 3(a) and (c); (g-m) are the test results of (f): (g) PPI by the 2D+t HLFs; (h) 2D+t FP-NLM filtered; (i) PPI by the 2D HLFs; (j-k) 2D FP-NLM filtered with parameter $\alpha_{2}=70(\mathrm{j})$ and $\alpha_{2}=90(\mathrm{k})$; (l) NLM filtered and (m) PBF filtered.

\begin{tabular}{|c|c|c|c|c|c|c|c|c|}
\hline$\sigma_{\varepsilon} /$ PSNR & NAD & TV & BF & WF & NLM & PBF & 2D FP-NLM & 2D+t FP-NLM \\
\hline \hline $20 / 21.91$ & 32.69 & 33.75 & 31.24 & 31.78 & 32.36 & 34.21 & $\mathbf{3 5 . 2 7}$ & 34.94 \\
$30 / 18.61$ & 31.30 & 31.89 & 27.90 & 30.14 & 30.22 & 32.13 & 32.88 & $\mathbf{3 3 . 7 6}$ \\
$40 / 16.43$ & 26.23 & 29.16 & 23.88 & 29.05 & 29.26 & 30.08 & 30.57 & $\mathbf{3 1 . 9 6}$ \\
\hline
\end{tabular}

Table 1: Performance comparisons of denoising algorithms.

\subsection{Test on real data}

We have further tested our 2D+t FP-NLM filter on a real live image sequence of EB1GFP, expressed in the Drosophila egg chamber where the microtubule cytoskeleton is complex and the imaging is challenging. The image sequence was collected on a 
DeltaVision imaging system over three Z planes. Fig. 6(a) is a frame from a single Z plane (low SNR image sequence) at time-point 115 (of 180) to be used for test. The EB1 foci (or particles) are barely visible. By average projection of the three $\mathrm{Z}$ planes, a corresponding high SNR image sequence in which the particles are visible can be obtained (the frame 115 is shown in Fig. 6(b)). The particles in the low SNR frame can be manually identified by comparing the corresponding tracks in the low/high SNR sequences.
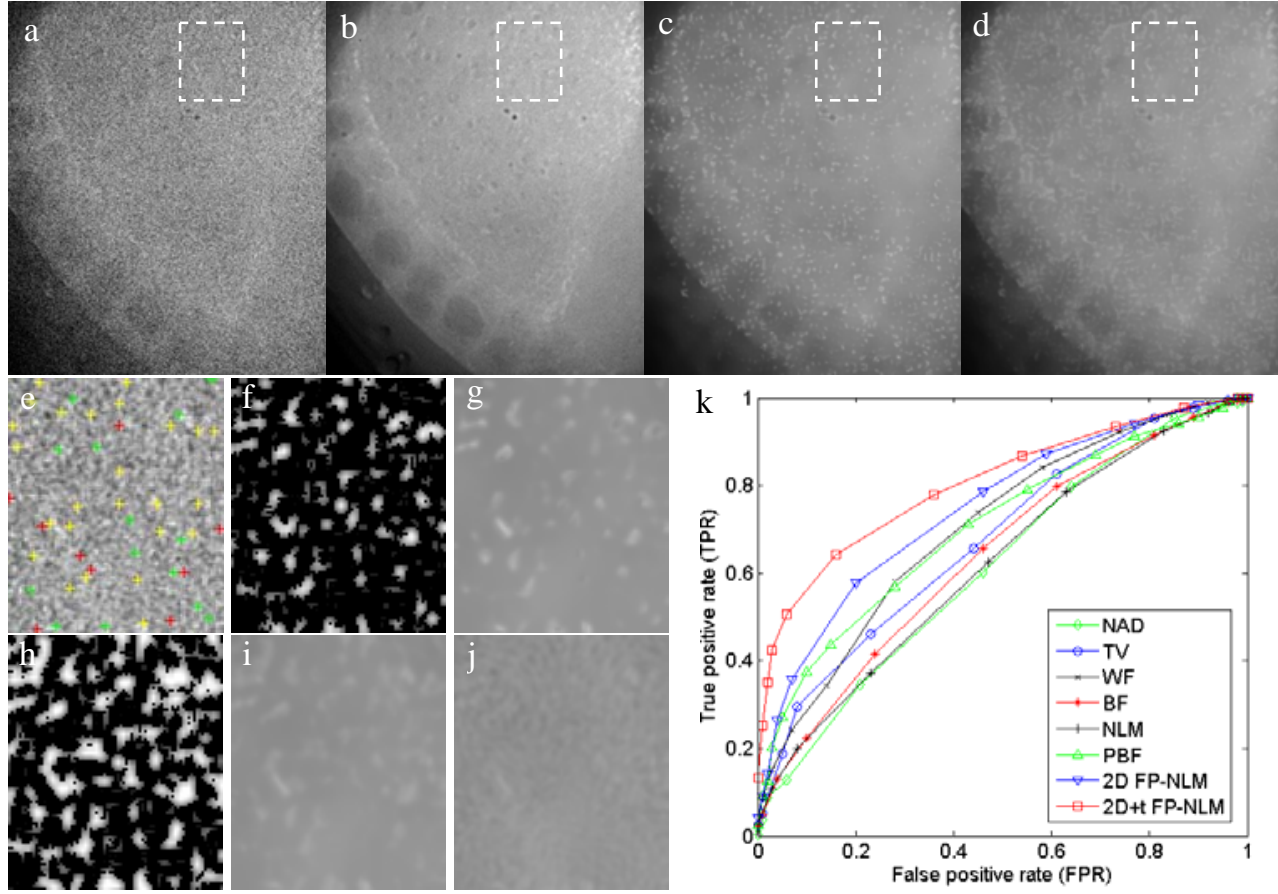

Figure 6. Test on time-lapse sequence. (a) a frame from low SNR sequence: single time point, single $\mathrm{Z}$ plane, and the noise standard deviation measured on the raw data is $\sigma=30$; (b) corresponding frame from good SNR sequence: $3 Z$ average projected; (c-d) denoising results from (a) by 2D+t FP-NLM (c) and 2D FP-NLM (d) filters, the threshold $\lambda=6.0, h=0.9 \sigma, g=\sigma / 150$ (c) and $g=\sigma / 70$ (d); (e-k) test on a subregion marked in (a): (e) manual identification of EB1 particles in the subregion, where the crosses indicate the locations of the particles, green: strong, yellow: weak, red: not visible; (f-g) PPI by 2D+t HLFs (f) and 2D+t FP-NLM filtered (g); (h-i) PPI by 2D HLFs (h) and 2D FP-NLM filtered (i); (j) NLM filtered; (k) ROC curves comparing denoising performance.

2D+t and 2D FP-NLM filtered results on the single frame Fig. 6(a) are shown in Fig. 6(c) and (d), where all the filtering parameters are chosen to be the same as in the synthetic test except that 4 different sizes of Haar windows are chosen from $7 \times 7$ to $13 \times 13$, because the smallest particles to be identified here are around $4 \times 4$ pixels. As can be seen, both the filters can significantly enhance the particles and smooth the noisy background. The particles in a subregion marked by a white box in Fig. 6(a) have been manually identified, as shown in Fig. 6(e). Due to the more accurate HLF classifications (compare Fig. 6(f) and (h) to (e)), 2D+t FP-NLM filter enhances better barely visible particles and generates fewer artefacts than 2D FP-NLM filter (compare Fig. 6(g) to (i)). For comparison, the NLM filtered result on the same subregion is shown in Fig. 6(j), where most of the particle-like 
features have been over-smoothed. Since an important task for denoising the images is to detect particles, we have computed the receiver operating characteristic (ROC) curves for the binarized denoised images as the threshold is varied. Here true positive rate (TPR) and false positive rate (FPR) are measured against a ground truth of the subregion, which is generated by the same method described in [12]. As seen from Fig. 6(k), the 2D+t FPNLM filtered image shows higher sensitivity to pick out true particle pixels than that generated by applying the 2D FP-NLM filter and other commonly used algorithms mentioned earlier. The results imply the 2D+t FP-NLM filter is a better algorithm to be used for particle detection in image sequences with high levels of noise contamination.

\section{Conclusion}

We have presented a new 2D+t FP-NLM filter for denoising images and improving detection of barely visible weak particles with high levels of noise contamination. Tests on synthetic and real biological image sequences show that the new algorithm achieves a better performance in enhancing particle contrast, smoothing background and minimizing artefacts. We note that while a simple CV motion model is used to construct the $2 \mathrm{D}+\mathrm{t}$ HLFs, it requires only 3 consecutive image frames in applications as discussed earlier and can apply as a good approximation to image sequences of varying particle velocity and direction if the variations are small within the 3 image frames. Moreover, since HLFs are obtained in Haar windows with different scales, they also give tolerance to variations of particle velocity and direction for their measurements. The CV motion model is therefore a simple but effective approach in extending the FP-NLM filter from 2D to 2D+t domain.

\section{References}

[1] D. J. Stephens and V. J. Allan. Light microscopy techniques for live cell imaging. Science, 300(5616):82-86, 2003.

[2] K. Jaqaman and D. Loerke et al. Robust single-particle tracking in live-cell time-lapse sequences. Nature Methods, 5(8):695-702, 2008.

[3] E. Meijering, I. Smal and G. Ganuser. Tracking in molecular bioimaging. IEEE Signal Processing Magazine, 23(3):46-53, 2006.

[4] J. Boulanger and C. Kervarann et al. Patch-based non-Local functional for denoising fluorescence microscopy image sequences. IEEE Trans. Medical Imaging. 29(2):442—454, 2010.

[5] I. F. Sbalzarini and P. Koumoutsakos. Feature point tracking and trajectory analysis for video imaging in cell biology. Journal of Structural Biology. 151(2):182—195, 2005.

[6] H. Leung and A. Young. Small target detection in clutter using recursive nonlinear prediction. IEEE Trans. Aerospace and Electronic Systems. 36(2):713-718, 2000.

[7] L. Yang, J. Yang and K. Yang. Adaptive detection for infrared small target under sea-sky complex background. Electronics Letters. 40(17):1083-1085, 2004.

[8] J. C. Curlander and R. N. McDonough. Synthetic aperture radar: systems and signal processing. New York: Wiley. 1991. 
[9] A. Buades, B. Coll and J. M. Morel. A review of image denoising algorithms, with a new one. Multiscale Modeling and Simulation. 4(2):490—530, 2005.

[10] A. Buades, B. Coll and J. M. Morel. Nonlocal image and movie denoising. International Journal of Computer Vision. 76(2):123-139, 2008.

[11] C. Kervrann and J. Boulanger. Local adaptivity to variable smoothness for exemplar-based image regularization and representation. International Journal of Computer Vision. 79(1):4569, 2008.

[12] L. Yang and R. Parton et al. An adaptive non-local means filter for denoising live-cell images and improving particle detection. Journal of Structural Biology (in press).

[13] P. Perona and J. Malik. Scale-space and edge detection using anisotropic diffusion. IEEE Trans. Pattern Analysis and Machine Intelligence. 12(7):629—639, 1990.

[14] L. I. Rudin, S. Osher and E. Fatemi. Nonlinear total variation based noise removal algorithms. Physica D. 60(1-4):259—268, 1992.

[15] C. Tomasi and R. Manduchi. Bilateral filtering for gray and color images. Proc. IEEE Int. Conf. Computer Vision. 839—846, 1998. 